\title{
THE INFLUENCE OF THE TYPE OF FILLING GAS ON THE RESPONSE OF IONISATION CHAMBERS TO A MIXED HIGH-ENERGY RADIATION FIELD
}

\author{
S. Mayer ${ }^{1, *}$, D. Forkel-Wirth ${ }^{2}$, M. Fuerstner ${ }^{2}$, S. Roesler ${ }^{2}$, C. Theis ${ }^{2}$ and H. Vincke ${ }^{2}$ \\ ${ }^{1}$ PSI, CH-5232 Villigen, Switzerland \\ ${ }^{2} \mathrm{CERN}, \mathrm{CH}-1211$ Geneva 23, Switzerland
}

\begin{abstract}
Radiation protection dosimetry in radiation fields behind the shielding of high-energy accelerators such as CERN is a challenging task and the quantitative understanding of the detector response used for dosimetry is essential. Measurements with ionisation chambers are a standard method to determine absorbed dose (in the detector material). For applications in mixed radiation fields, ionisation chambers are often also calibrated in terms of ambient dose equivalent at conventional reference radiation fields. The response of a given ionisation chamber to the various particle types of a complex high-energy radiation field in terms of ambient dose equivalent depends of course on the materials used for the construction and the chamber gas used. This paper will present results of computational studies simulating the exposure of high-pressure ionisation chambers filled with different types of gases to the radiation field at CERN's CERN-EU high-energy reference field facility. At this facility complex high-energy radiation fields, similar to those produced by cosmic rays at flight altitudes, are produced. The particle fluence and spectra calculated with FLUKA Monte Carlo simulations have been benchmarked in several measurements. The results can be used to optimise the response of ionisation chambers for the measurement of ambient dose equivalent in high-energy mixed radiation fields.
\end{abstract}

\section{INTRODUCTION}

Several radiation components (photons, neutrons, different types of charged particles), covering a wide range of energies, up to the $\mathrm{GeV}$ region, contribute to the total dose equivalent in the radiation field behind the shielding of high-energy accelerators such as those at CERN. Radiation protection dosimetry in such complex radiation fields is therefore a challenging task and a quantitative understanding of the detector response used for dosimetry is required.

Measurements with ionisation chambers are a standard method to determine absorbed dose (in the detector material). For applications in mixed radiation fields, ionisation chambers are often also calibrated in terms of ambient dose equivalent in calibration fields realised by radioactive sources. The response of a given ionisation chamber to the various particle types of a complex high-energy radiation field in terms of ambient dose equivalent depends of course on the materials used for the construction and the chamber's gas filling.

This paper compares the results of computational studies simulating the exposure of Centronics IG5 high-pressure ionisation chambers filled with different types of gases to the high-energy, mixed radiation field at CERN's CERN-EU high-energy reference field (CERF) facility. First simulation studies were restricted to the use of pure hydrogen and pure argon gas-fillings. For the obtained results

\footnotetext{
*Corresponding author: sabine.mayer@psi.ch
}

benchmark measurements at CERF showed very good agreement ${ }^{(1)}$. Subsequent to these successful investigations new simulations were carried out in order to extend the studies to commercially available chambers of the same type using other gas-fillings such as pure nitrogen and a mixture of argon/nitrogen gas (A17N3, 1.7 MPa Ar/ 0.3 MPa N 2 ). These different fillings are superior compared to pure argon-fillings in strongly pulsed fields where recombination effects are not negligible. This is because the collection efficiency of an ionisation chamber can be corrected for volume recombination effects according to a model of Boag ${ }^{(2,3)}$, provided that the current is carried entirely by positive and negative ions. That means that electrons attach themselves to gas molecules and that no appreciable part of the current is carried by free electrons. In a gas such as argon, where electron attachment does not occur, the correction by Boag is not valid. This limitation of argon could be observed in a measurement campaign in strongly pulsed fields reported in the work of Forkel-Wirth et al. ${ }^{(4)}$.

\section{RESPONSE STUDIES OF IONISATION CHAMBERS IN MIXED RADIATION FIELDS}

\section{Response to various particle types}

The responses of Centronics IG5 ionisation chambers with an active volume of $5.2 \mathrm{dm}^{3}$ and a filling-pressure of $2 \mathrm{MPa}$ were simulated, using four different gas-fillings: hydrogen (H20), argon (A20), nitrogen (N20) and a mixture of argon/nitrogen 
Table 1. $W$-values used in this study as given in ICRUreport $31^{(7)}$.

\begin{tabular}{llccc}
\hline & \multicolumn{4}{c}{$W$-value (eV) } \\
\cline { 2 - 5 } & Argon & A17N3 & Nitrogen & Hydrogen \\
\hline Photons & 26.4 & 28.0 & 34.8 & 36.5 \\
Neutrons & 26.6 & 28.0 & 34.8 & 36.6 \\
Protons & 26.6 & 28.0 & 36.7 & 36.6 \\
Pions, & 26.6 & 28.0 & 34.8 & 36.6 \\
$\quad$ Electrons, & & & & \\
$\quad$ Muons & & & & \\
\hline
\end{tabular}

(A17N3). For all calculations the radiation transport code FLUKA (H20, A20: FLUKA version 2002; N20, A17N3: FLUKA version 2003) $)^{(5,6)}$ was used.

The primary quantity calculated by FLUKA is energy deposition, which was recorded for the active volume of the chamber. Dividing the energy deposition by the mean energy which is required to produce an ion pair ( $W$-value), yields the created charge in the chamber. The $W$-value is defined and extensively discussed in the ICRU report $31^{(7)}$. It is dependent on the gas type, the type of radiation and its energy. However, it was shown that the energy dependence is weak and, thus, can be approximated in most cases by a constant value for the respective gas type and for specific particle types. The $W$-values adopted in this study are listed in Table 1 . Empirically determined values could be found for pure gas-fillings in ICRU-report $31^{(7)}$, whereas for the A17N3-filling the case of a binary gas mixture was assumed.

According to the ICRU-report 31 for a large number of binary gas mixtures the $W$-value, denoted by $W_{i j}$ with the gas components $i$ and $j$, is given by

$$
\frac{1}{W_{i j}}=\left(\frac{1}{W_{i}}-\frac{1}{W_{j}}\right) \cdot Z_{i j}+\frac{1}{W_{j}}
$$

where

$$
Z_{i j}=\frac{P_{i}}{P_{i}+a_{i j} P_{j}}
$$

$W_{i}, W_{j} \ldots W$-values for the pure components $\rightarrow W_{\mathrm{Ar}}=26.4 \mathrm{eV}, W_{\mathrm{N}}=34.8 \mathrm{eV}$

$P_{i}, P_{j} \ldots$ partial pressures of the two components $\rightarrow P_{\mathrm{i}}=1.7 \mathrm{MPa}, P_{j}=0.3 \mathrm{MPa}$

$a_{i j} \ldots$ empirical constant, following the suggestion of ICRU-report $31^{(7)}$ it can be assumed as the ratio $a_{i j}=S_{j} / S_{i}$

$S_{i}, S_{j} \ldots$ stopping power

Due to the fact that the ratio of the stopping powers $S_{\mathrm{N}} / S_{\mathrm{Ar}}$ that were taken from Berger et al. ${ }^{(8)}$, is a function of energy, one obtains a $W$-function rather than a constant. However, evaluating the function in the range from $1 \mathrm{keV}$ up to $1 \mathrm{GeV}$ shows only weak energy dependence. Since the standard deviation from the average value of $28.0 \mathrm{eV}$ was found to be only $0.5 \%$ the $W$-function is approximated by a constant value of $W_{\mathrm{A} 17 \mathrm{~N} 3}=28.0 \mathrm{eV}$.

The simulations which assume a parallel beam of mono-energetic particles of a certain type focused on the major radiation components encountered in mixed radiation fields such as neutrons, photons, electrons, protons, pions and muons. Further components of the mixed radiation field were neglected because of their minor contribution to the total dose. In the case of photons the measurements that were performed using X-ray sources were simulated by sampling the respective energy spectra from standardized distributions ${ }^{(9)}$.

In the present paper the responses of the ionisation chambers are shown exemplarily for neutrons and photons. In Figure 1 the photon response in terms of created charge per energy released in unit mass of air is presented for the different gas-fillings of the IG5 chamber. The applied fluence to air kerma conversion factors were taken from ICRP $74^{(10)}$. For pure argon- and hydrogen-filled chambers measurement results ${ }^{(1)}$ were available and are included in the figure. The respective statistical uncertainties of the calculations are so small that the error bars are smaller than the data point symbols in all subsequent figures.

Moreover, in Figure 2 the respective response to photons expressed in terms of created charge per unit ambient dose equivalent is illustrated. The values were obtained by the application of

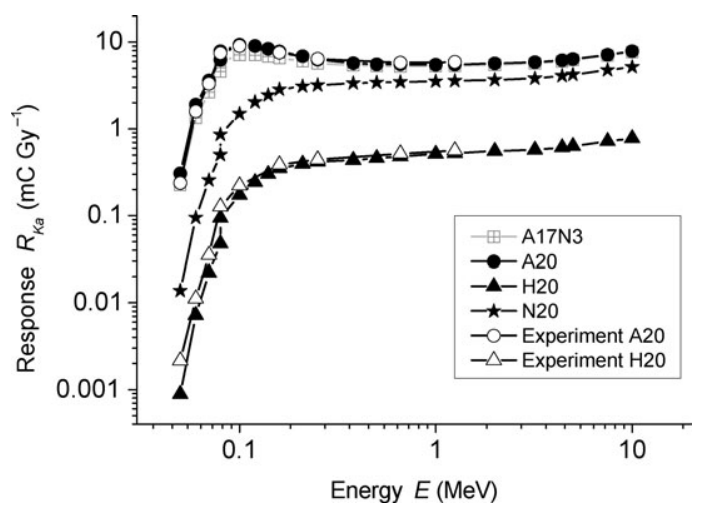

Figure 1. Photon response in terms of created charge per energy released in unit mass of air presented for the different gas-fillings of the IG5 chamber. Fluence to air kerma conversion factors are taken from ICRP74 ${ }^{(10)}$. In addition, experimental results obtained by exposing the chamber to various calibration sources are shown. 


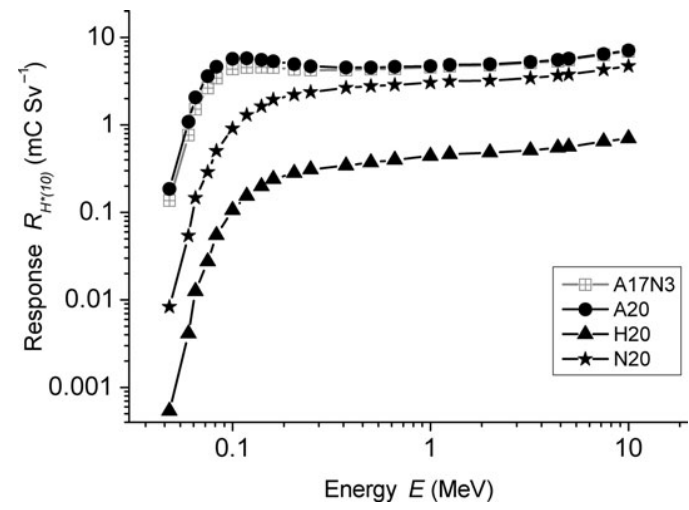

Figure 2. Photon response functions of the IG5 chamber for different gas-fillings expressed in terms of created charge per unit ambient dose equivalent. Fluence to ambient dose equivalent conversion factors are taken from Pelliccioni ${ }^{(12)}$.

fluence-to-ambient dose equivalent conversion factors according to Pellicioni ${ }^{(12)}$. Similar to the calculations for photons the response functions for neutrons in terms of charge per unit ambient dose equivalent were obtained. The calculations were carried out for neutrons within an energy range from thermal energies up to $5 \mathrm{GeV}$. In the case of neutrons elastic and inelastic reactions below 19.6 MeV are simulated by FLUKA using tabulated cross-sections. Consequently, the binning in the response function is corresponding to this energy range. The calculated results for the respective gas fillings are shown in Figure 3. Note that the response

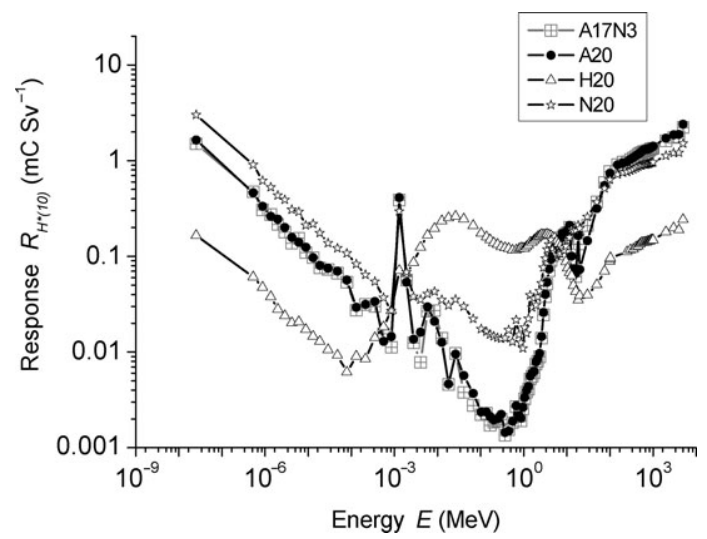

Figure 3. Neutron response functions of the IG5 chamber for different gas-fillings. The response is expressed in created charge per unit ambient dose equivalent. Fluence to ambient dose equivalent conversion factors are taken from Pelliccioni ${ }^{(12)}$. of the A17N3 gas-filling does not always lie between the response of pure argon and pure nitrogen gasfillings. This can be explained by the fact that the $W$-value is assumed to be different for the various gases and it appears as a scaling factor on the calculated results. Additionally, it is mentioned in ICRUreport $31^{(7)}$, that a very small amount added to noble gases can change the ionisation characteristics considerably. One should, therefore, still examine the behaviour of the chambers experimentally.

\section{Response to mixed radiation fields}

The response of the chambers was then investigated in a typical high-energy field such as the CERN-EU high-energy reference field (CERF) ${ }^{(13)}$ facility. The facility offers various reference positions behind $80 \mathrm{~cm}$ thick concrete shielding that are well characterised with FLUKA simulations ${ }^{(14)}$. In Figure 4 the typical fluence spectra simulated for different particle types at a reference position behind the concrete shield are given as an example.

As can be seen in the figure, the field is composed mainly of neutrons and photons ranging from thermal energies up to GeV. Thus, the ambient dose equivalent is strongly dominated by neutrons ( $\sim 85 \%$ of the dose equivalent), the rest is due to photons.

Folding the results of the chamber response with the appropriate particle fluence spectra at CERF yielded the created charge within the active volume of the detector per primary particle. Figure 5 illustrates the relative contribution of each particle type to the total charge per primary particle for the reference position CS2. The contribution of kaons was neglected in the figure because of their minor contribution to the total charge.

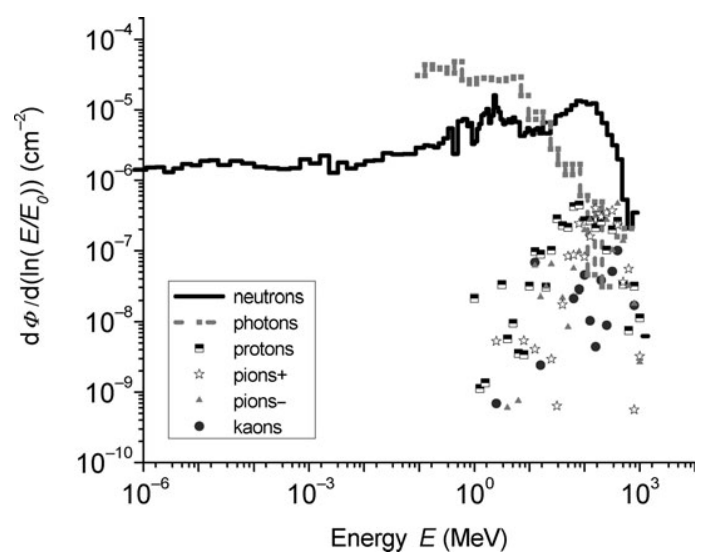

Figure 4. Particle fluence spectra calculated with FLUKA behind the concrete shielding at position CS2 at CERF ${ }^{(14)}$. 
INFLUENCE OF FILLING GAS ON RESPONSE

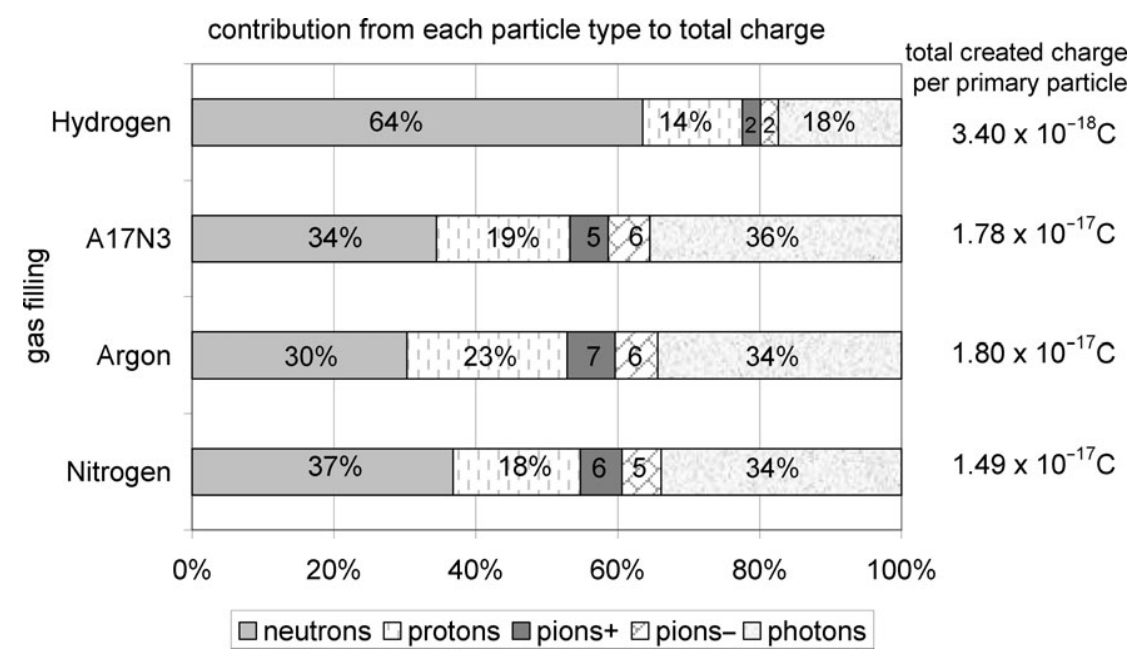

Figure 5. Relative contribution from each particle type to the total created charge for a mixed radiation field encountered at the reference position CS2 at CERF.

The simulations show that in the CERF reference field, the total created charge per primary particle of the argon- or nitrogen-filled chamber is approximately five times higher than that of a hydrogen-filled chamber. This would indicate that in a CERF-like field the argon- or nitrogen-filled chamber would be the preferred instrument regarding response in terms of total created charge. However, the major contribution to the expected ambient dose equivalent outside the shielding originates from neutrons as neutrons have the highest radiation quality factor. Therefore, it is crucial to know the response of the detectors to neutrons to be able to choose the one which measures the corresponding dose contribution best. One can see from Figure 5 that the hydrogen chamber is the one with the highest relative sensitivity to neutrons regarding total charge.

\section{CONCLUSION}

The influence of the type of filling gas on the response of ionisation chambers was simulated for a typical high-energy radiation field using the parameters of the CERF field. For two of the filling gases former measurement campaigns validated the simulations. These FLUKA simulations were then extended to other commercially available filling gases such as nitrogen and a mixture of argon and nitrogen. The simulations showed that the response to the various particle types is very similar between the argon-filled and nitrogen-filled chamber. However, the advantage of nitrogen-filled IG5 chambers is that they allow for a correction of recombination effects which is not possible for argon-filled chambers. The detailed exploration of the different responses to the various particle types permits a qualified choice for the future use of the investigated chambers.

\section{REFERENCES}

1. Theis, C., Rettig, M., Roesler, S. and Vincke, H. Simulation and experimental verification of the response functions of Centronic high-pressure ionisation chambers. Technical Note, CERN-SC-2004-23-RP-TN (2004).

2. Boag, J. W. Ionization measurements at very high intensities. Br. J. Radiol. 23, 601-611 (1950).

3. International Commission on Radiation Units and Measurements. Dosimetry of pulsed radiation. ICRU Report 34 (1982).

4. Forkel-Wirth, D., Mayer, S., Menzel, H. G., Muller, A., Otto, T., Pangallo, M., Perrin, D., Rettig, M., Roesler, S. and Scibile, L. et al. Performance requirements for monitoring pulsed, mixed radiation fields around high energy accelerators, Proceedings of EPAC 2004, Lucerne, P. 147 (2004).

5. Fassò, A., Ferrari, A., Ranft, J. and Sala, P. R. FLUKA: a multi-particle transport code. CERN yellow report, INFN/TC_05/11, SLAC-R-773 (2005).

6. Fassò, A., Ferrari, A., Roesler, S., Sala, P. R., Battistoni, G., Cerutti, F., Gadioli, E., Garzelli, M. V., Ballarini, F. and Ottolenghi, A. et al. The physics models of FLUKA: status and recent developments. Computing in high energy and nuclear physics 2003 conference (CHEP2003), La Jolla, CA, USA, , March 24-28, 2003, (paper MOMT005) Conf C0303241, arXiv:hep-ph/0306267 (2003).

7. International commission on radiation units and measurements. Average energy required to produce an ion pair. ICRU report 31, 7910 Woodmont Avenue, Washington, D. C. 20014, USA (1979). 


\section{S. MAYER ET AL.}

8. Berger, M. J., Coursey, J. S. and Zucker, M. A. Stopping-power and range tables for electrons, protons, and helium ions, physical reference data, NIST. Available on http://physics.nist.gov/PhysRefData/ Star/Text/contents.html (2005).

9. Ankerhold, U. Catalogue of X-ray spectra and their characteristic data ISO and DIN radiation qualities, therapy and diagnostic radiation qualities, unfiltered $X$-ray spectra. PTB Bericht PTB-Dos-34 (2000).

10. International Commission for Radiological Protection. Conversion Coefficients for Use in Radiological Protection, ICRP 74 (Oxford (UK): Pergamon Press) (1997).

11. Hess, A. Détermination de la réponse de chambres d'ionisation prévues pour la surveillance du LHC.
CERN/Haute Ecole Spécialisée du Suisse Occidentale, (2002).

12. Pelliccioni, M. Overview of fluence-to-effective dose and fluence-to-ambient dose equivalent conversion coefficients for high energy radiation calculated using the FLUKA Code. Radiat. Prot. Dosim. 88, 279-297 (2000).

13. Mitaroff, A. and Silari, M. The CERN-EU high-energy reference field (CERF) facility for dosimetry at commercial flight altitudes and in space. Radiat. Prot. Dosim. 102 (1), 7-22 (2002).

14. Theis, C., Roesler, S. and Vincke, H. Comparison of the simulation and measurements of the response of Centronic high-pressure ionisation chambers to the mixed radiation field of the CERF facility. Technical Note, CERN-SC-2004-24-RP-TN (2004). 